\title{
Use of Sputum Eosinophil Count in differentiating Bronchial Asthma from COPD
}

\author{
Dr.R.Narmadhalakshmimd ${ }^{1}$, Dr.B.Prabakar.Md ${ }^{2}$, \\ ${ }^{1 .}$ Associate Professor Of Medicine, Government Chengalpattu Medical College Hospital,Chengalpattu. \\ ${ }^{2}$.Asst Professor, Dept Of General Medicine, Government Chengalpattumedical College Hospital, \\ Chengalpattu.
}

\section{Introduction}

Bronchial Asthma and COPD are airway disorders and form a major part of the Physician workload. Both the diseases present as airway obstruction producing wheeze. Though Pulmonary Function Test and other modalities of investigation are available to help us differentiating between these two airway disorders , they may not be available always and everywhere. Hence the need for a test that is useful, less expensive and reproducible one, which helps to differentiate between Asthma and COPD.The Study conducted at Chengalpattu.Medical college and Hospital In the period of January 2015 to November 2015 study group consist of fifty patient each Bronchial asthma, COPD and control.

\section{Aim}

1.To assess the validity of Sputum Eosinophil count in the diagnosis of Asthma.

2. To know if the Sputum Eosinophil count can be utilized in differentiation of Bronchial Asthma from COPD.

Three groups of patients were selected.

\section{Methods And Materials.}

1. Bronchial asthmatics - subjected to PFT pre and post bronchodilator ; patients having a reversibility of > $20 \%$ were selected.

2. COPD - subjected to PFT pre and post bronchodilator ; patients with reversibility $<20 \%$ and $\mathrm{h} / \mathrm{o}$ smoking were included.

3. Controls - patients with cough $<2$ wks with $\mathrm{h} / \mathrm{o}$ no wheeze, with a normal PFT were selected as Controls.

All the patients were subjected to the following investigations.

Blood total, differential count

Blood eosinophil count

Chest Xray

Sputum culture and sensitivity

Sputum eosinophil percentage.

\section{Exclusion criteria;}

1. Sputum AFB positive

2. Sputum culture positive

3. CXR Old PT Sequalae

Sputum by spontaneous cough or by induced cough with nebulized normal saline were collected in containers; they were smeared on glass slides and eosin, hematoxylin staining was done. Stained slides were handed over to the Pathologist for sputum eosinophil study; he was not revealed about the identity of the slides. Salivary contamination was determined using percentage of squamous cells among nucleated cells. If squamous cells were $>10 \%$ of nucleated cells, then salivary contamination was high and the slide was discarded.

100 nucleated cells were counted and the percentage of eosinophils among them was calculated. High power and oil immersion fields were utilized for the study.

\section{Results}

Sputum eosinophil percentage of $>3 \%$ was taken as positive result and less than that as negative result.

Age :
\begin{tabular}{|l|l|l|}
\hline Disease & Age (mean) & SD \\
\hline Bronchial asthma & 35.56 & 12.94 \\
\hline COPD & 54.38 & 7.89 \\
\hline Controls & 42.76 & 16.68 \\
\hline
\end{tabular}




Sex :
\begin{tabular}{|l|l|l|}
\hline Disease & Female & Male \\
\hline Bronchial asthma & $23(46 \%)$ & $27(54 \%)$ \\
\hline COPD & $11(22 \%)$ & $39(78 \%)$ \\
\hline Controls & $23(46 \%)$ & $27(54 \%)$ \\
\hline
\end{tabular}

Sputum eosinophil percentage (SEP) $>3 \%$ in Asthma, COPD and Controls.

\begin{tabular}{|l|l|l|l|}
\hline SEP & Asthma & COPD & Controls \\
\hline$>3 \%$ & 38 & 21 & 2 \\
\hline$<3 \%$ & 12 & 29 & 48 \\
\hline
\end{tabular}

$\mathrm{X} 253.77 \mathrm{P}$ value 0.001 Sputum eosinophil percentage in asthma and controls X2 6.73 P value 0.009 Sputum eosinophil percentage in asthma and COPD X2 11.95; P value 0.01 Significant P values shows that sputum eosinophil count can be taken up for differentiating Bronchial asthma and COPD ; and can be used in diagnosis of Asthma. The 95\% confidence interval levels of sputum eosinophil count in Asthmatics is $61.83 \%$ 86.94\%; For COPD it is $28.195-56.79 \%$ infers that it is a significant test in Asthmatics than in COPD.

\section{Discussion}

Huster et al compared the validity of different tests in adult asthmatics and found that methacholine airwayresponsiveness and a sputum differential, eosinophil test are useful tests in asthma.

Ruth Greene et $\mathrm{al}^{1}$ had discussed about the usefulness of sputum eosinophil count in the management of Asthma. Bouwmik etal ${ }^{2}$ study did not show any difference between induced cough and spontaneous cough sputum samples.

In COPD aptients $40 \%$ of people showed sputum eosinophil $>3 \%$. Brightling etal ${ }^{3}$ showed that sputum eosinophils in COPD suggests steroid responsiveness.

Rosi etal ${ }^{4}$ states that sputum eosinophils has a good diagnostic accuracy in differentiating asthma fro non asthma. Rhonchi etal ${ }^{4}$ says that sputum eosinophil predominate in asthma than in COPD where neutrophils predominate.

\section{Conclusion}

1 sputum eosinophil percentage $>3 \%$ can be used as one of the tools in diagnosis of asthma.

2. it can be used to differentiate from COPD.

\section{References}

[1]. Ruth H Green et al; Asthma exacerbations and sputum eosinophil counts: a randomized controlled trai,lThe Lancet Nov 2002; Vol,360;

[2]. A Bhowmik et al- comparison of spontaneous and induced sputum for investigation airway inflammation in chronic obstructive pulmonary disease

[3]. Brigthling et al- sputum eosinophilia\& short term response to prednisolone in chronic obstructive pulmonary disease- arandomized controlled trial-Lancet;2000;Oct 356:1480-5

[4]. Rosi E Ronchi et al-Diagnostic accyracy of sputum outcomes in chronic stable asthma-clini exp Allergy 2000 Apr 30:577-84. 